\title{
Comparative determination of HIV-1 co-receptor tropism by Enhanced Sensitivity Trofile, gp120 V3-loop RNA and DNA genotyping
}

\author{
Mattia CF Prosperi*1, Laura Bracciale1, Massimiliano Fabbiani1', Simona Di Giambenedetto1, Francesca Razzolini2, \\ Genny Meini², Manuela Colafigli' ${ }^{1}$ Angela Marzocchetti ${ }^{1}$, Roberto Cauda' ${ }^{1}$, Maurizio Zazzi² and Andrea De Luca'1,3
}

\begin{abstract}
Background: Trofile is the prospectively validated HIV-1 tropism assay. Its use is limited by high costs, long turnaround time, and inability to test patients with very low or undetectable viremia. We aimed at assessing the efficiency of population genotypic assays based on gp120 V3-loop sequencing for the determination of tropism in plasma viral RNA and in whole-blood viral DNA. Contemporary and follow-up plasma and whole-blood samples from patients undergoing tropism testing via the enhanced sensitivity Trofile (ESTA) were collected. Clinical and clonal geno2pheno $_{\text {[coreceptor] }}(\mathrm{G} 2 \mathrm{P}$ ) models at 10\% and at optimised 5.7\% false positive rate cutoff were evaluated using viral DNA and RNA samples, compared against each other and ESTA, using Cohen's kappa, phylogenetic analysis, and area under the receiver operating characteristic (AUROC).

Results: Both clinical and clonal G2P (with different false positive rates) showed good performances in predicting the ESTA outcome (for V3 RNA-based clinical G2P at 10\% false positive rate $A U R O C=0.83$, sensitivity $=90 \%$, specificity $=$ $75 \%$ ). The rate of agreement between DNA- and RNA-based clinical G2P was fair (kappa $=0.74, p<0.0001$ ), and DNAbased clinical G2P accurately predicted the plasma ESTA (AUROC $=0.86$ ). Significant differences in the viral populations were detected when comparing inter/intra patient diversity of viral DNA with RNA sequences.

Conclusions: Plasma HIV RNA or whole-blood HIV DNA V3-loop sequencing interpreted with clinical G2P is cheap and can be a good surrogate for ESTA. Although there may be differences among viral RNA and DNA populations in the same host, DNA-based G2P may be used as an indication of viral tropism in patients with undetectable plasma viremia.
\end{abstract}

\section{Background}

Maraviroc (MVC) is the first CCR5 antagonist approved for the treatment of HIV-1 infection [1] following the demonstration of its virological efficacy in treatmentexperienced patients $[2,3]$. There is reasonable expectation that MVC or other CCR5-antagonists can be even better administered to treatment-naïve patients due to a higher prevalence of CCR5-tropic (R5) HIV-1 in this population as compared to more advanced patients [4]. Due to the lack of virologic activity against CXCR4-tropic (X4) virus [5], the administration of MVC is subject to the verification of an $\mathrm{R} 5$ virus population in the candidate

* Correspondence: ahnven@yahoo.it

1 Infectious Diseases Clinic, Catholic University of Sacred Heart, Rome, Italy Full list of author information is available at the end of the article patient. The enhanced sensitivity Trofile assay (ESTA) is the current gold standard phenotypic method for the determination of co-receptor tropism for the replicating viral population (i.e. plasma RNA), although other inhouse or commercial tests are available, some of which use peripheral blood mononuclear cell (PBMC DNA) $[6,7]$. The drawbacks of any phenotypic test include high costs, long turn-around time, and reduced efficiency in patients with low viremia. For this reason, there is a demand for a fast and cheap HIV-1 tropism assay to fully exploit CCR5 antagonists as a treatment option in clinical routine $[8,9]$.

Given that most of the determinants of viral co-receptor tropism are based on polymorphisms of the third hypervariable region (V3) of the gp120, an alternative to 
the phenotypic approach is the usage of machine learning tools based on viral genotypic information. So called insilico or virtual phenotype models may be indeed convenient for clinical practice due to the reduction of costs and turn-around time. During the recent years, several prediction models have been studied, from the first simple rule based on the polymorphisms at V3 codons 11 and 25, to the position specific scoring matrices (PSSM), neural networks, support vector machines, random forests and logistic models [10-20]. Some of these studies identified additional factors possibly impacting viral tropism, such as viral subtype and CD4 cell counts $[14,16,20]$. Comparisons among genotypic and phenotypic tests have been carried out. The genotypic geno2pheno ${ }_{\text {[coreceptor] }}$ system [16] was compared with the first generation Trofile and the TRT phenotypic assays [21], showing $86.5 \%$ and $79.7 \%$ concordance, respectively. A study comparing the predictive performance of geno2pheno $_{\text {[coreceptor] }}$, PSSM [12] and other methods against the first-generation Trofile assay, concluded that current default implementations of co-receptor prediction algorithms were inadequate for predicting CXCR4 co-receptor usage in clinical samples, due to inability to detect low-level X4 virus [22]. Another study found the concordance among genotype-based predictors and firstgeneration Trofile ${ }^{\circ}$ being as high as 91\% [23]. Variable performance of in-silico models was shown when considering non-B HIV-1 variants [24-26].

Concerning the clinical validation of phenotypic assays, another recent work focused on the performance of the Trofile test in predicting the virological response to a short-term maraviroc exposure in HIV-infected patients [27]. Concomitantly, a few attempts to unveil mutational patterns associated to selection by CCR 5 antagonists or resistance have been carried out $[28,29]$, but the frequency and rate at which maraviroc resistance mutations emerge are not yet known.

The most awaited information is how in-silico tropism prediction models predict virological response to CCR5antagonists, particularly when genotypic and phenotypic results disagree. In fact, although the ESTA should detect lower amounts of $\mathrm{X} 4$ virus compared to bulk genotyping, the X4 level threshold compromising in vivo CCR5antagonist activity in vivo is currently unknown. In addition, ESTA cannot be performed at low or undetectable viral load (VL), while HIV-1 DNA genotyping can be easily performed in such cases; and this information, if adequately validated, might be easily employed for guiding treatment switches to CCR5 antagonists in virologically suppressed patients, due to toxicity or simplification issues. Finally, genotyping can also be used to detect HIV1 mutations selected by MVC and possibly decreasing its effectiveness. Our study aimed at evaluating the accuracy of HIV-1 co-receptor tropism prediction by viral RNA and DNA genotyping, as well as the selection of $\mathrm{V} 3$ mutants in MVC-failing individuals.

\section{Methods \\ Patients}

Contemporary plasma and whole-blood samples were prospectively collected from HIV-infected patients followed up at a single centre of the Infectious Diseases Clinic of Catholic University of Sacred Heart in Rome, Italy, all failing antiretroviral treatment and potentially candidates for treatment with a CCR5-antagonists, in the period between November 2007 and July 2009 ( $\mathrm{n}=55$ ). Some of these patients underwent tropism testing via the ESTA ( $\mathrm{n}=51)$. Follow-up plasma and whole-blood samples from these patients were also collected, regardless of MVC treatment.

\section{Viral amplification and sequencing}

Plasma RNA and whole-blood DNA were obtained from citrated blood by spin column extraction (Qiagen, Hilden, Germany). Plasma underwent 1-hour centrifugation at $23,000 \times \mathrm{g}$ at $4^{\circ} \mathrm{C}$ to concentrate virus prior to extraction. Whole blood was used without pre-processing steps. A 419-bp region encompassing the HIV-1 gp120 V3 domain was amplified by (RT)-PCR and sequenced in both strands by infrared-labelled primers on a Licor IR2 system. Plasma RNA was reverse transcribed with primer P151 (5'-CTACTTTATATTTATATAATTCAYTTCTC-3', coordinates 7661-7689 in the reference $\mathrm{HXB} 2$ genome). The reverse transcription reaction was run for 30 minutes at $37^{\circ} \mathrm{C}$ and included 10 $\mu \mathrm{L}$ of RNA extract, $50 \mathrm{mM}$ Tris- $\mathrm{HCl}\left(\mathrm{pH} 8.3\right.$ at $\left.25^{\circ} \mathrm{C}\right), 75$ $\mathrm{mM} \mathrm{KCl,} 10 \mathrm{mM}$ DTT, $3 \mathrm{mM} \mathrm{MgCl} 2,200 \mu \mathrm{M}$ each dNTP, 200 U ImProm II RT (Promega), 20 U RNasin (Promega) and 5 pmol primer P151. The cDNA obtained (one third of the reverse transcription mixture) or blood DNA ( $1 \mu \mathrm{g})$ extracted from whole-blood was amplified by a nested PCR protocol using primer P150 (5'-AATGTCAGCACAGTACAATGYACACAT-3', 6945-6971) and P151 in the outer amplification step and primer LR33 (5'-CAGTACAATGTACACATGGAAT-3', 6955-6976) and LR34 (5'-GAAAAATTCCCCTCCACAATT-3', 7353-7373) in the inner amplification step. Both outer and inner PCR mixtures contained $50 \mathrm{mM}$ Tris- $\mathrm{HCl}\left(\mathrm{pH} 9.0\right.$ at $25^{\circ} \mathrm{C}$ ), 50 $\mathrm{mM} \mathrm{KCl}, 2 \mathrm{mM} \mathrm{MgCl}_{2}, 200 \mu \mathrm{M}$ each dNTP, $1.25 \mathrm{U}$ GoTaq polymerase (Promega) and 8 pmol each primer. The cycling profile was 20 seconds at $52^{\circ} \mathrm{C}, 40$ seconds at $72^{\circ} \mathrm{C}$ and 30 seconds at $94^{\circ} \mathrm{C}$ for both steps but the number of cycles was 25 in the outer PCR and 32 in the inner PCR. The final product was sequenced by the IRD700- 
labelled sense primer IR25 (5'-GCTGTTAAATGGCAGTCTAGCAGAA-3', 7011-7035) and the IRD800labelled antisense primer IR77 (5'-GAAAAATTCTCCTCYACAATTRA-3', 7351-7373) using the DYEnamic Direct Cycle Sequencing kit with 7-deaza-dGTP (GE Healthcare). Sequence contigs were assembled by the DNASTAR SeqMan II version 5.07 module. Only one PCR product per sample was subjected to standard population sequencing, expected to allow detection of minority species contributing at least $20 \%$ of the whole virus quasispecies.

\section{Sequence analysis: tropism prediction and its evaluation}

The geno2pheno ${ }_{\text {[coreceptor] }}$ in-silico genotypic tropism prediction system was employed, using both clinical (requiring VL, nadir CD4 and CD8 count) and clonal interpretations at $10 \%$ false positive rate (FPR) [17], and by considering a clonal interpretation at the optimised cutoff of 5.75\% FPR, based on analysis of clinical data from MOTIVATE and MERIT studies [30,31]. Concordances among geno2pheno predictors and ESTA were assessed by Cohen's kappa statistic [32]. The predictive ability of the systems was evaluated by receiver operating characteristic (ROC) analysis [33,34].

The distance between nucleotide V3 sequences was calculated by the maximum composite likelihood [35], over a multiple alignment obtained via MUSCLE [36]. Phylogenetic analysis was performed using the MEGA 4 software [37], estimating tree and branch support with a bootstrapped neighbour-joining method.

Analysis of selection of env mutations by MVC exposure was performed by considering patients with a RNA sequence before (the closest to the start date) and after (considering the latest after at least one month of therapy) MVC initiation. Amino acid mutations were extracted by local pairwise alignments against the HXB2 reference sequence. Fisher's exact test on frequency counts of individual mutations and pre- post-MVC strata was executed; obtained p-values were also corrected with Benjamini-Hochberg procedure.

The $\mathrm{R}$ mathematical programming suite was used to perform all statistical analyses [38].

\section{Results}

\section{Study population and samples}

We processed 178 samples (99 plasma RNA and 79 whole-blood DNA) and successfully amplified 155 samples [78 (78.8\%) plasma RNA and 77 (97.5\%) whole-blood DNA] from 55 patients. By stratifying for contemporary $\mathrm{VL}$, the rate of successful sequencing from RNA was $40 \%$, $88.2 \%$, and $96.2 \%$ at $<=50 \mathrm{cp} / \mathrm{ml}, 51-500 \mathrm{cp} / \mathrm{ml}$, and $>500$ $\mathrm{cp} / \mathrm{ml}$ respectively. The rate of successful sequencing from DNA was $95.1 \%, 100 \%$, and $100 \%$ at $<=50 \mathrm{cp} / \mathrm{ml}$, $50-500 \mathrm{cp} / \mathrm{ml}$, and $>500 \mathrm{cp} / \mathrm{ml}$, respectively.
Fifty-one of the 55 patients had their baseline plasma tested by ESTA, and 28 were subsequently administered MVC. Patients' baseline characteristics are summarized in Table 1.

In a cross sectional crude analysis, using dichotomized ESTA results (R5-tropic versus X4- plus dual/mixedtropic), we did not find any significant association with contemporary patients characteristics, except for the time from HIV-1 diagnosis [median (IQR) 16 years (1318) for R5, and 18 years (17-22) for $\mathrm{X} 4$ or dual/mixedtropic isolates $\mathrm{p}=0.007$ ], and nadir $\mathrm{CD} 4$ count [median (IQR) 138 cells $/ \mathrm{mm} 3$ (31-200) for R5 and 14 cells $/ \mathrm{mm} 3$ (6-41) for X4 or dual/mixed-tropic isolates $\mathrm{p}=0.05]$.

\section{Comparison between geno2 pheno $_{\text {[coreceptor] }}$ classification of plasma HIV-1 RNA, DNA V3 sequences and ESTA tropism results}

We first compared the prediction performance of geno2pheno versus the ESTA result. For this analysis, we excluded samples not reportable by ESTA. To match geno2pheno categories, the dual/mixed virus classification by ESTA was pooled with the $\mathrm{X} 4$ virus classification. Figure 1 depicts ROC plots with the performance of two RNA-based geno2pheno models predicting the ESTA X4 or dual/mixed tropism. Using nucleotide V3 loop sequences obtained from RNA samples contemporary to ESTA testing $(\mathrm{n}=35)$, the resulting area under the ROC curve (AUROC) was 0.83 for geno2pheno clinical interpretation, 0.67 for geno2pheno clonal interpretation at $10 \%$ FPR, and 0.75 using the optimised $5.75 \%$ FPR cutoff. When comparing differences in AUROC with respect to the clinical interpretation reference, neither the clonal interpretation at 10\% FPR nor that at 5.75\% FPR cutoff exhibited a statistically significant lower area $(\mathrm{p}=0.17$ and $\mathrm{p}=0.48$, respectively).

As a second performance test, we obtained geno2pheno tropism predictions for $\mathrm{V} 3$ sequences obtained from DNA samples $(\mathrm{n}=17$, with 16 sequences from patients with contemporary RNA genotyping). The resulting prediction of ESTA X4-D/M tropism showed an AUROC of 0.86 using geno2pheno clinical interpretation, 0.69 using the geno2pheno clonal interpretation at $10 \%$ FPR, and 0.76 using the optimised 5.75\% FPR cutoff (Figure 2). We did not find significant differences in AUROC when comparing either the clonal interpretation $(\mathrm{p}=$ $0.34)$ at $10 \%$ FPR or that at $5.75 \%$ FPR cutoff $(\mathrm{p}=0.58)$ against the clinical geno2pheno interpretation at $10 \%$ FPR.

Table 2 shows accuracy, sensitivity and specificity of the clinical, and clonal interpretation at $10 \%$ or $5.75 \%$ FPR cutoff of RNA/DNA geno2pheno interpretation modes in predicting ESTA X4-D/M-tropism. 
Table 1: Patients' characteristics at the time of ESTA

\begin{tabular}{|c|c|c|c|c|}
\hline \multicolumn{2}{|c|}{ No. of patients with available ESTA result } & \multicolumn{3}{|l|}{51} \\
\hline \multirow{4}{*}{\multicolumn{2}{|c|}{ ESTA results, n (\%) }} & \multicolumn{2}{|l|}{ CCR5-tropic } & $31(61 \%)$ \\
\hline & & \multicolumn{2}{|l|}{ Dual/Mixed-tropic } & $12(23 \%)$ \\
\hline & & \multicolumn{2}{|l|}{ CXCR4-tropic } & $1(2 \%)$ \\
\hline & & \multicolumn{2}{|l|}{ Not Reportable } & $7(14 \%)$ \\
\hline \multicolumn{2}{|l|}{ Descriptive } & total & CCR5-tropic & Dual/Mixed/CXCR4-tropic \\
\hline \multicolumn{2}{|l|}{ Female gender, n (\%) } & $16(31 \%)$ & $12(39 \%)$ & $4(31 \%)$ \\
\hline \multicolumn{2}{|l|}{ Mean age (SD) } & $45(9)$ & $46(8)$ & $40(12)$ \\
\hline \multicolumn{2}{|c|}{ Median time from HIV-1 diagnosis, years (IQR) } & $17(14-19)$ & $16(13-18)$ & $18(17-22)$ \\
\hline \multicolumn{2}{|l|}{ Italian nationality, n (\%) } & 48 (94\%) & $29(93 \%)$ & $12(92 \%)$ \\
\hline \multirow[t]{4}{*}{ Risk Factor, n (\%) } & Heterosexual & $15(29 \%)$ & $11(35 \%)$ & $3(23 \%)$ \\
\hline & Homosexual/Bisexual & $17(33 \%)$ & $11(35 \%)$ & $4(31 \%)$ \\
\hline & IDU & $14(27 \%)$ & $7(23 \%)$ & $3(23 \%)$ \\
\hline & Other/Unknown & $5(10 \%)$ & $2(6 \%)$ & $3(23 \%)$ \\
\hline \multirow[t]{3}{*}{ CDC stage, n (\%) } & A & $16(32 \%)$ & $11(35 \%)$ & $3(25 \%)$ \\
\hline & B & $10(20 \%)$ & $9(29 \%)$ & $1(8 \%)$ \\
\hline & C & $24(48 \%)$ & $11(35 \%)$ & $8(67 \%)$ \\
\hline \multicolumn{2}{|c|}{ History of mono-dual NRTI therapy, $\mathrm{n}(\%)$} & $37(72 \%)$ & $24(77 \%)$ & $10(77 \%)$ \\
\hline \multicolumn{2}{|c|}{ Median duration of ART exposure, years (IQR) } & $14(11-16)$ & $13(11-16)$ & $13(11-15)$ \\
\hline \multicolumn{2}{|c|}{$\begin{array}{l}\text { No. of drug switches (any change or interruption) } \\
\text { experienced (IQR) }\end{array}$} & $13(9-16)$ & $13(9-15)$ & $11(7-15)$ \\
\hline \multirow[t]{3}{*}{$\begin{array}{l}\text { Patients previously exposed to } \\
\text { drug class, } \mathrm{n}(\%)\end{array}$} & NRTI & $50(98 \%)$ & $31(100 \%)$ & $13(100 \%)$ \\
\hline & NNRTI & $44(86 \%)$ & $29(93 \%)$ & $10(77 \%)$ \\
\hline & Unboosted PI & $49(96 \%)$ & $30(97 \%)$ & $13(100 \%)$ \\
\hline
\end{tabular}


Table 1: Patients' characteristics at the time of ESTA (Continued)

\begin{tabular}{lccc}
\hline \multicolumn{1}{c}{ Boosted PI } & $35(69 \%)$ & $22(71 \%)$ & $8(61 \%)$ \\
\hline CD4 count, median cells/mm3 (IQR) & $334(182-535)$ & $387(214-464)$ & $211(198-518)$ \\
HIV-1 RNA load, median log10 cp/ml (IQR) & $3.66(2.55-4.30)$ & $3.9(3.6-4.1)$ & $4.2(4.0-4.8)$ \\
CD4 count at nadir, median cells/mm3 (IQR) & $64(18-191)$ & $138(31-200)$ & $14(6-41)$ \\
HIV-1 RNA load at zenith, median Log10 cp/ml (IQR) & $5.3(4.6-5.7)$ & $5.5(4.8-5.7)$ & $5.4(4.5-5.6)$ \\
\hline
\end{tabular}

\section{Comparison of HIV-1 V3 RNA and DNA sequence population and their inferred coreceptor tropisms}

The median intrapatient distance among HIV RNA or DNA sequences was, as expected, significantly smaller than the interpatient distance (see Additional files 1 and 2). However, the intrapatient variability with RNA (0.007) or with DNA (0.015) sequences was significantly lower than the intrapatient variability between RNA and DNA sequences (0.023), suggesting that the HIV-1 V3 DNA and RNA populations may contribute different information and complement each other in a patient. Phylogenetic analysis revealed that clusters of sequences with high support values (bootstrap $>90 \%$ ) corresponded to sequences drawn from the same patients, and there were no clusters composed of sequences from different patients. Clusters were preferentially (but not exclusively) composed of either paired RNA or DNA samples (phylogenetic tree is available as Additional file 1).

We next investigated whether a certain degree of intrapatient diversity between DNA and RNA populations results in a different co-receptor tropism prediction. When comparing geno2pheno clinical interpretation based on paired HIV-1 V3 DNA-RNA sequences obtained from 29 distinct patients, we observed 35/40 (87.5\%) concordant predictions, $3 / 40$ (7.5\%) false positives, and 2/40 (5\%) false negatives, using RNA-predicted $\mathrm{X} 4$-tropism as the reference outcome. The kappa statistics yielded a strength of agreement of 0.74 (95\% CI: 0.530.95), with a Fisher's p-value $<0.0001$. The AUROC obtained by predicting the geno2pheno RNA tropism from contemporary DNA sequences was 0.875 .

\section{Viro-immunological follow up}

Out of 28 patients with an R5-tropic virus by ESTA subsequently starting MVC, 22 had an available virological follow up at 12 weeks. All patients had a VL below $50 \mathrm{cp} /$ $\mathrm{ml}$, except two patients with 120 and $292 \mathrm{cp} / \mathrm{ml}$. Virological follow-up at 24 weeks was available for 19 patients. All of these had a VL below $50 \mathrm{cp} / \mathrm{ml}$, except two patients (different from those at three months) with a VL of 2,003 and $88 \mathrm{cp} / \mathrm{ml}$.

Immunological follow-up at 12 weeks was available for 20 out of the 28 patients that started MVC. The median
(IQR) CD4 increase was 84 (range -9 to 165 ) cells/mm3. A Wilcoxon test showed that this increase from baseline was statistically significant $(\mathrm{p}=0.019)$. After 24 weeks of therapy, immunological follow-up was available for 19 patients. The median (IQR) CD4 increase was 46 (-9 to 143) cells $/ \mathrm{mm} 3(\mathrm{p}=0.053)$.

\section{Evolution of V3 sequences during MVC therapy}

Finally, we executed statistical tests for difference in proportions by looking at the whole set of mutations retrieved in the RNA samples with respect to HIV-1 HXB2 envelope reference, grouping sequences in preand post-MVC initiation ( $\mathrm{n}=18,9$ sequences pre-MVC, 9 post-MVC). Of the 9 pre-MVC sequences (all R5 by ESTA), 4 (44\%) were classified as X4 by clinical geno2pheno interpretation, with FPR of $4.8 \%, 7.8 \%, 0.2 \%$, and $0.8 \%$. Tropism prediction did not change for any patient when considering post-MVC samples. No substitution was significantly associated with MVC exposure by correcting test statistics with Benjamini Hochberg (all p-values $=1$ ) nor retaining raw unadjusted p-values; nonetheless a deletion at position 354, mutation $355 \mathrm{~K}$, 369L, and 259Q (all in the V3 loop) showed an increase in prevalence after MVC initiation. Figure 3 depicts mutations detected in the V3 loop in pre- and post-MVC samples with the lowest unadjusted p-values.

\section{Discussion}

In the present study, viral tropism prediction by the HIV1 gp120 V3-loop RNA genotype-based geno2pheno ${ }_{\text {[core- }}$ ceptor] interpretation in treatment-experienced subjects proved to be a valid alternative to ESTA tropism, yielding an AUROC of 0.83 using the clinical interpretation. The clonal interpretation showed a lower AUROC, although the difference with the clinical interpretation showed only a trend, probably due to the limited sample size. The geno2pheno ${ }_{\text {[coreceptor] }}$ website suggests that only the clonal interpretation should be used for treatment-experienced patients, since the clinical system was trained only on treatment-naïve patients. However, our results show that the clinical interpretation is better (at least not inferior) than the clonal interpretation even in treatment-experienced patients. It is important to highlight the fact that 


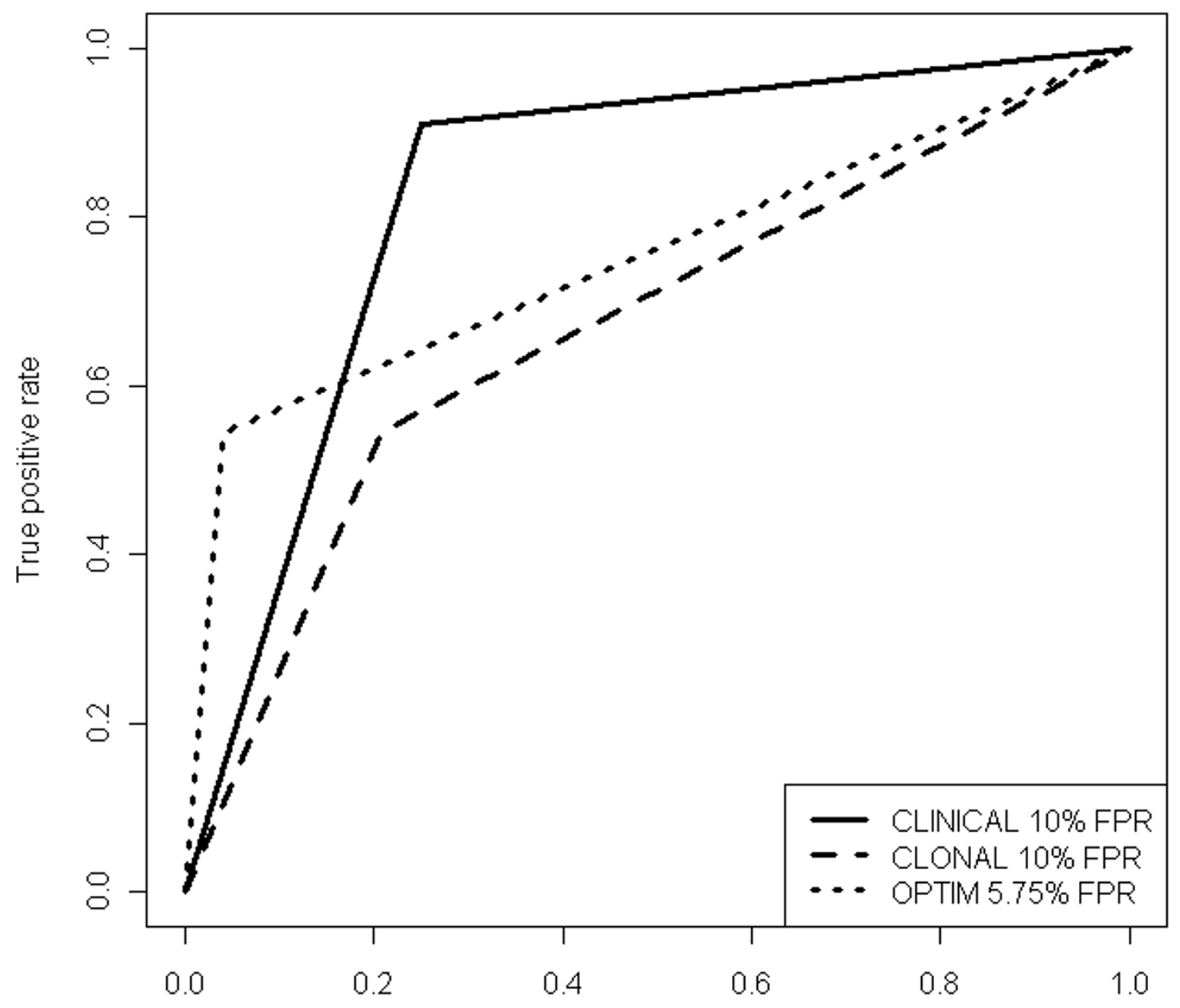

False positive rate

Figure 1 Area under the ROC curves (AUROC) comparing predictions from RNA samples using geno2pheno clinical (AUROC $=0.83$ ) at $10 \%$ FPR, geno2pheno clonal (AUROC $=0.67$ ) at $10 \% \mathrm{FPR}$, and clonal at $5.75 \%$ FPR cutoff (AUROC $=0.75$ ) interpretation modes versus the ESTA result $(n=35)$.

the clinical interpretation needs contemporary VL, nadir CD4 and CD8 information in order to work properly. Interestingly, the clinical mode improved sensitivity much more than specificity with respect to the clonal mode. The sensitivity of V3 RNA genotyping followed by clonal geno2pheno ${ }_{[\text {coreceptor] }}$ interpretation has been recently shown to increase by testing multiple aliquots of the plasma RNA extract [39], likely as a consequence of stochastic fluctuations of a minority X4 populations. Although repeated testing has not yet been established as a standard procedure, it is expected that its implementation would result in a higher concordance between tro- pism results obtained by $\mathrm{V} 3$ genotyping and the reference ESTA.

Another area under investigation is the optimal FPR to use with geno2pheno ${ }_{\text {[coreceptor] }}$ as a cut-off for classification of R5 and X4-D/M viruses. Recent retrospective analysis of the MERIT trial data indicated that the most accurate FPR cutoff may be in the range from $2 \%$ to $5.75 \%$, when triplicate PCR testing and fully automated sequencing is used [30]. However, there is currently no consensus on which cut-off to use in clinical practice. As for any classification test aimed at orienting a clinical intervention, the trade-off between specificity and sensi- 


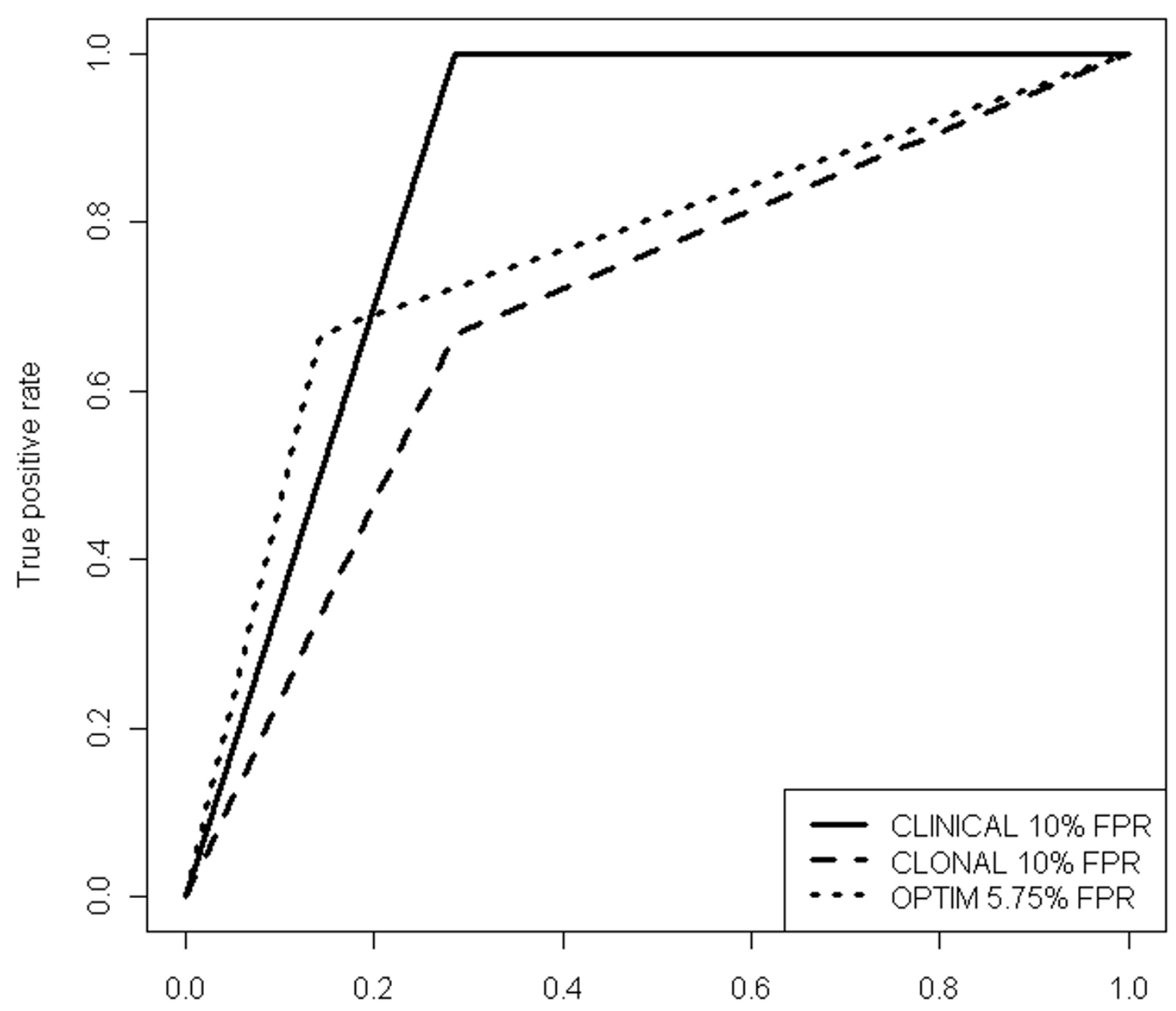

False positive rate

Figure 2 Area under the ROC curve (AUROC) comparing predictions of geno2pheno clinical (AUROC $=0.86$ ) at $10 \%$ FPR, geno2pheno clonal (AUROC $=0.69$ ) at $10 \% \mathrm{FPR}$, and clonal at $5.75 \% \mathrm{FPR}$ cutoff ( $A U R O C=0.76$ ) interpretation modes versus the ESTA result, using sequences obtained from contemporary DNA samples $(n=17)$.

tivity must be taken into account. A higher cut-off such as the $10 \%$ FPR was originally proposed [17] and is still recommended by the expert panel developing and maintaining the geno2pheno ${ }_{[\text {coreceptor] }}$ system (see the German recommendations [40]). Using a higher cut-off translates into a conservative attitude, i.e. a lower probability to treat with maraviroc patients who may not benefit from it, at the expense of a higher probability not to treat patients who may benefit from the drug. It remains to be established whether this lower cut-off can be clinically more convenient in the genotypic screening of the gen- eral HIV patient population candidate to treatment with maraviroc.

One major advantage with genotyping is that even patients with non-reportable ESTA can be given prediction of tropism. By definition, ESTA is subject to a larger proportion of failures with respect to genotype due to virus polymorphisms invalidating the cloning procedure and an inherently lower rate of reverse transcription of a far larger virus genome region. Moreover, our efficiency of RNA genotyping at VL between 50 and $500 \mathrm{cp} / \mathrm{ml}$ (where ESTA is not even attempted) was $88 \%$, whilst that of DNA genotyping was $100 \%$ at these viral loads. 
Table 2: Performance evaluation of ESTA X4-dual/mixed tropism prediction using geno2pheno clinical and clonal at $10 \%$ FPR, or the clonal at the optimised 5.75\% FPR interpretation of contemporary viral gp120 V3 DNA or RNA genotyping

\begin{tabular}{|c|c|c|c|c|c|}
\hline Viral sample & $\begin{array}{l}\text { geno2pheno } \\
\text { interpretation } \\
\text { mode (FPR) }\end{array}$ & AUROC & accuracy & sensitivity & specificity \\
\hline \multirow[t]{3}{*}{ plasma RNA ( $n=35)$} & Clinical (10\%) & 0.83 & $80.0 \%$ & $90.9 \%$ & $75.0 \%$ \\
\hline & Clonal (10\%) & 0.67 & $71.4 \%$ & $54.5 \%$ & $79.2 \%$ \\
\hline & $\begin{array}{l}\text { Clonal optim. } \\
(5.75 \%)\end{array}$ & 0.75 & $82.9 \%$ & $54.5 \%$ & $95.8 \%$ \\
\hline \multirow[t]{3}{*}{ whole-blood DNA $(n=17)$} & Clinical (10\%) & 0.86 & $76.5 \%$ & $100 \%$ & $71.4 \%$ \\
\hline & Clonal (10\%) & 0.69 & $70.6 \%$ & $66.7 \%$ & $71.4 \%$ \\
\hline & $\begin{array}{l}\text { Clonal optim. } \\
(5.75 \%)\end{array}$ & 0.76 & $82.3 \%$ & $66.7 \%$ & $85.7 \%$ \\
\hline
\end{tabular}

Interestingly, in-silico tropism prediction using wholeblood DNA genotyping may be a solution when considering treatment switch to a CCR5-antagonists for patients with undetectable viral load. The perspective for treatment switches in these patients may be attractive, because of the good tolerability of MVC and because patients that are not at a late stage of disease are more likely to harbour a CCR5- rather than a CXCR4-tropic virus [4]. In these cases ESTA cannot be used, and RNA genotyping is often not sufficiently efficient. The performance of DNA-based clinical geno2pheno in predicting the ESTA result was comparable to that of RNA-based clinical geno2pheno $($ AUROC $=0.86)$. Thus, the use of V3 DNA sequence data for predicting co-receptor tropism definitely warrants further investigation as an appealing alternative to RNA.

As expected, we found some differences when comparing paired DNA and RNA sequences, consistent with the notion that the archived population may not correspond to the most prevalent virus in plasma, whose source are the productively infected cells. However, when comparing DNA and RNA tropism prediction by looking at contemporary samples, the degree of agreement was good, implying that such minor differences may not commonly translate into inappropriate indications. It remains to be established whether an X4 virus population detected in PBMC DNA in the context of R5 virus in plasma RNA can impair response to maraviroc. Although reported on a limited number of cases, this did not appear to be the case in the French GenoTropism study [41,42].

\section{Conclusion}

HIV-1 tropism determination via plasma viral V3 RNA genotyping coupled with geno2pheno interpretation may represent a valid alternative to ESTA. The clinical validation of genotypic determination of viral tropism has been recently performed using retrospective samples from the MOTIVATE study [40] as well as in the GenoTropism study where the genotypic tropism test was able to predict response to maraviroc even in the group of patients with an R5 virus population as detected by standard Trofile $[41,42]$. As shown here, RNA tropism genotyping carries the advantage of a higher efficiency of tropism determination in patients with low copy number detectable viral loads. In addition to that, in perspective, DNAbased tropism prediction could be used in patients with undetectable VL who are candidates for treatment simplification/switch to a CCR5-antagonist. This option can support a more effective use of this class of agents at earlier stages when the probability of harbouring an R5 virus population is maximal. However, further investigations to unveil the evolutionary relationships between DNA and RNA populations are advisable before DNA genotyping can be indicated in clinical practice. In this context, ultradeep sequencing studies may be appropriate to dissect the dynamics and role of DNA and RNA minority variants $[43,44]$. Most importantly, clinical validation of the use of HIV-1 genotyping, particularly with proviral DNA, for tropism assignment is also required before its widespread implementation. 


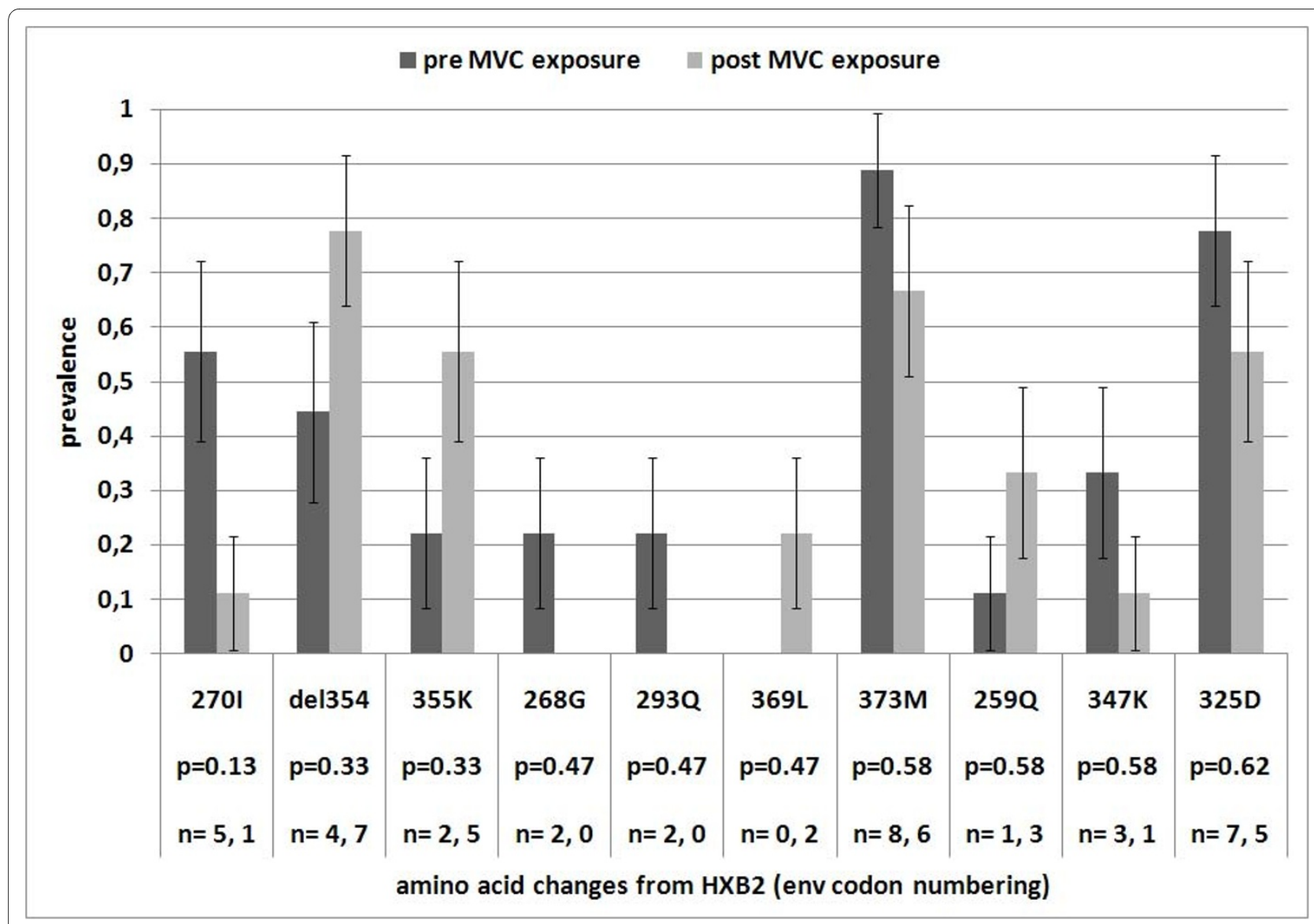

Figure 3 Prevalence of mutations (with respect to HIV-1 HXB2) in the V3 loop from RNA samples, stratified by MVC exposure ( $n=18,9$ sequences pre-MVC, 9 post-MVC). Mutations are shown in decreasing order by unadjusted $p$-value obtained from a Fisher's test comparing pre- vs. post-MVC prevalence

\section{Additional material}

Additional file 1 Evolutionary relationships of 155 V3 sequences obtained from 51 patients at different time points joining RNA and DNA samples + 1 outgroup (HIV-1 group J, V3 loop). Sequences are labelled by DNA/RNA type, by sampling date (the number before the type), and by patient's identifier (first one or two numbers). When considering all the 155 viral DNA/RNA V3 sequences obtained from all the 55 patients, the median (IQR) distance among all samples was 0.126 (0.101-0.159). The median (IQR) interpatient RNA-RNA distance was $0.133(0.110-0.166)(\mathrm{n}=$ 2,946 pairs) and DNA-DNA $(n=2,874)$ distance was $0.121(0.095-0.152)$. The median (IQR) intrapatient RNA-RNA $(n=56)$, DNA-DNA $(n=52)$ and paired RNA-DNA $(n=120)$ distances were 0.007 (0.000-0.017), $0.015(0.007-0.031)$ and 0.023 (0.015-0.031), respectively, with a Kruskal's p-value $<0.0001$.

Additional file 2 Detailed classifications of tropism by different geno2pheno modes and ESTA on our data sets.

\section{Competing interests}

Maurizio Zazzi has received recent research funding from Pfizer; served as a consultant for Abbott Molecular, Boehringer Ingelheim, Gilead Sciences, and Janssen; and served on speakers' bureaus for Abbott, Bristol-Myers Squibb, Merck, and Pfizer.

Andrea De Luca received speakers honoraria, served as consultant or participated in advisory boards for GlaxoSmithKline, Gilead, Bristol-Myers Squibb, Abbott Virology, Tibotec-Janssen, Siemens Diagnostics and Monogram Biosciences.
Roberto Cauda has attended advisory boards or has been a consultant for Glaxo-SmithKline, Gilead, Bristol-Myers Squibb, Boehringer Ingelheim, Abbott Virology, Novartis, Pfizer, Schering-plough, and Merck Sharp \& Dohme.

All other authors declare no competing interests.

\section{Authors' contributions}

MCFP assisted with manuscript writing and statistical analyses; LB, MF, SDG and MC assisted with patients' care and data acquisition; FR, GM and AM assisted with laboratory assays; RC, MZ and ADL assisted with manuscript revision and research group leading. All authors read and approved the final manuscript.

\section{Acknowledgements}

This work has been partly supported by EU-funded projects DynaNets (grant \#233847) and CHAIN (grant \#223131).

\section{Author Details}

IInfectious Diseases Clinic, Catholic University of Sacred Heart, Rome, Italy, 2Molecular Biology Department, University of Siena, Siena, Italy and 3 Infectious Diseases Unit, University Hospital of Siena, Siena, Italy

Received: 9 March 2010 Accepted: 30 June 2010 Published: 30 June 2010

\section{References}

1. Sayana S, Khanlou H: Maraviroc: a new CCR5 antagonist. Expert Rev Anti Infect Ther 2009, 7(1):9-19.

2. Heera J, Ive P, Botes M, Dejesus E, Mayer H, Goodrich J, Clumeck N, Cooper DA, Walmsley S, Craig C, Reeves J, van der Ryst E, Saag M: The MERIT 
study of maraviroc in antiretroviral-naive patients with R5 HIV-1: 96week results [abstract]. 5th IAS Conference on HIV Pathogenesis, Treatment and Prevention. Cape Town, South Africa 2009.

3. Fätkenheuer $G$, Nelson M, Lazzarin A, Konourina I, Hoepelman Al, Lampiris H, Hirschel B, Tebas P, Raffi F, Trottier B, Bellos N, Saag M, Cooper DA, Westby M, Tawadrous M, Sullivan JF, Ridgway C, Dunne MW, Felstead S, Mayer $\mathrm{H}$, van der Ryst E, MOTIVATE 1 and MOTIVATE 2 Study Teams: Subgroup analyses of maraviroc in previously treated R5 HIV-1 infection. N Engl J Med 2008, 359(14):1442-55.

4. Vandekerckhove L, Verhofstede C, Vogelaers D: Maraviroc: perspectives for use in antiretroviral-naive HIV-1-infected patients. J Antimicrob Chemother 2009, 63(6):1087-96.

5. Saag M, Goodrich J, Fätkenheuer G, Clotet B, Clumeck N, Sullivan J, Westby M, van der Ryst E, Mayer H, A4001029 Study Group: A double-blind, placebo-controlled trial of maraviroc in treatment-experienced patients infected with non-R5 HIV-1. J Infect Dis 2009, 199(11):1638-47.

6. Braun P, Wiesmann F: Phenotypic assays for the determination of coreceptor tropism in HIV-1 infected individuals. Eur J Med Res 2007, 12(9):463-72.

7. Coakley E, Reeves JD, Huang W, Mangas-Ruiz M, Maurer I, Harskamp AM, Gupta S, Lie Y, Petropoulos CJ, Schuitemaker H, van't Wout AB: Comparison of human immunodeficiency virus type 1 tropism profiles in clinical samples by the Trofile and MT-2 assays. Antimicrob Agents Chemother 2009, 53(11):4686-93.

8. Rose JD, Rhea AM, Weber J, Quiñones-Mateu ME: Current tests to evaluate HIV-1 coreceptor tropism. Curr Opin HIV AIDS 2009, 4(2):136-42.

9. Soriano V, Perno CF, Kaiser R, Calvez V, Gatell JM, di Perri G, Pillay D, Rockstroh J, Geretti AM: When and how to use maraviroc in HIV-infected patients. AIDS 2009, 23(18):2377-85

10. De Jong JJ, De Ronde A, Keulen W, Tersmette M, Goudsmit J: Minimal requirements for the human immunodeficiency virus type $1 \mathrm{~V} 3$ domain to support the syncytium-inducing phenotype: analysis by single amino acid substitution. J Virol 1992, 66(11):6777-6780

11. Resch W, Hoffman N, Swanstrom R: Improved success of phenotype prediction of the human immunodeficiency virus type 1 from envelope variable loop 3 sequence using neural networks. Virology 2001, 288:51-62.

12. Jensen MA, van't Wout AB: Predicting HIV-1 coreceptor usage with sequence analysis. AIDS Rev 2003, 5:104-112.

13. Pillai S, Good B, Richman D, Corbeil J: A new perspective on V3 phenotype prediction. AIDS Res Hum Retroviruses 2003, 19:145-149.

14. Sander O, Sing T, Sommer I, Low AJ, Cheung PK, Harrigan PR, Lengauer T, Domingues FS: Structural descriptors of gp120 V3 loop for the prediction of HIV-1 coreceptor usage. PLoS Comput Biol 2007, 3(3):e58.

15. Xu S, Huang $X, X u H$, Zhang $C$ : Improved prediction of coreceptor usage and phenotype of HIV-1 based on combined features of V3 loop sequence using random forest. J Clin Microbio/ 2007, 45(5):441-6.

16. Sing T, Low AJ, Beerenwinkel N, Sander O, Cheung PK, Domingues FS, Büch J, Däumer M, Kaiser R, Lengauer T, Harrigan PR: Predicting HIV coreceptor usage on the basis of genetic and clinical covariates. Antivir Ther 2007, 12(7):1097-106.

17. Lengauer T, Sander O, Sierra S, Thielen A, Kaiser R: Bioinformatics prediction of HIV coreceptor usage. Nat Biotechnol 2007. 25(12):1407-10.

18. Lamers SL, Salemi M, McGrath MS, Fogel GB: Prediction of R5, X4, and R5X4 HIV-1 coreceptor usage with evolved neural networks. IEEE/ACM Trans Comput Biol Bioinform 2008, 5(2):291-300.

19. Boisvert S, Marchand M, Laviolette F, Corbeil J: HIV-1 coreceptor usage prediction without multiple alignments: an application of string kernels. Retrovirology 2008, 5:110.

20. Prosperi MC, Fanti I, Ulivi G, Micarelli A, De Luca A, Zazzi M: Robust supervised and unsupervised statistical learning for HIV type 1 coreceptor usage analysis. AIDS Res Hum Retroviruses 2009, 25(3):305-14.

21. Skrabal K, Low AJ, Dong W, Sing T, Cheung PK, Mammano F, Harrigan PR: Determining human immunodeficiency virus coreceptor use in a clinical setting: degree of correlation between two phenotypic assays and a bioinformatic model. J Clin Microbio/ 2007, 45(2):279-84.

22. Low AJ, Dong W, Chan D, Sing T, Swanstrom R, Jensen M, Pillai S, Good B, Harrigan PR: Current V3 genotyping algorithms are inadequate for predicting X4 co-receptor usage in clinical isolates. AIDS 2007, 21(14):F17-24.
23. Raymond S, Delobel P, Mavigner M, Cazabat M, Souyris C, Sandres-Sauné K, Cuzin L, Marchou B, Massip P, Izopet J: Correlation between genotypic predictions based on $\mathrm{V} 3$ sequences and phenotypic determination of HIV-1 tropism. AIDS 2008, 22(14):F11-6.

24. Garrido C, Roulet V, Chueca N, Poveda E, Aguilera A, Skrabal K, Zahonero N, Carlos S, García F, Faudon JL, Soriano V, de Mendoza C: Evaluation of eight different bioinformatics tools to predict viral tropism in different human immunodeficiency virus type 1 subtypes. J Clin Microbio/ 2008, 46(3):887-91.

25. Raymond S, Delobel P, Mavigner M, Ferradini L, Cazabat M, Souyris C, Sandres-Sauné K, Pasquier C, Marchou B, Massip P, Izopet J: Prediction of HIV type 1 subtype $C$ tropism by genotypic algorithms built from subtype B viruses. J Acquir Immune Defic Syndr 2010, 53(2):167-75.

26. Raymond S, Delobel P, Mavigner M, Cazabat M, Souyris C, Encinas S, Sandres-Sauné K, Pasquier C, Marchou B, Massip P, Izopet J: Genotypic prediction of human immunodeficiency virus type 1 CRF02-AG tropism. J Clin Microbiol 2009, 47(7):2292-4.

27. Genebat M, Ruiz-Mateos E, León JA, González-Serna A, Pulido I, Rivas I, Ferrando-Martínez S, Sánchez B, Muñoz-Fernández MA, Leal M: Correlation between the Trofile test and virological response to a short-term maraviroc exposure in HIV-infected patients. J Antimicrob Chemother 2009, 64(4):845-9.

28. Anastassopoulou CG, Ketas TJ, Klasse PJ, Moore JP: Resistance to CCR5 inhibitors caused by sequence changes in the fusion peptide of HIV-1 gp41. Proc Natl Acad Sci USA 2009, 106(13):5318-23.

29. Moore JP, Kuritzkes DR: A pièce de resistance: how HIV-1 escapes small molecule CCR5 inhibitors. Curr Opin HIV AIDS 2009, 4(2):118-24.

30. McGovern R, Dong W, Zhong X, Knapp D, Thielen A, Chapman D, Lewis M, James I, Valdez H, Harrigan R: Population-based Sequencing of the V3loop Is Comparable to the Enhanced Sensitivity Trofile Assay in Predicting Virologic Response to Maraviroc of Treatment-naï i²ve Patients in the MERIT Trial [abstract]. 17th Conference on Retroviruses and Opportunistic Infections. San Francisco, USA 2010

31. Harrigan PR, McGovern R, Dong W, Thielen A, Jensen M, Mo T, Chapman D, Lewis M, James I, Ellery S, Heera J, Valdez H: Screening for HIV Tropism using Population based V3 Genotypic Analysis: a Retrospective Virologic Outcome Analysis Using Stored Plasma Screening Samples from the MOTIVATE Studies of Maraviroc in Treatment Experienced Patients [abstract]. 5th IAS Conference on HIV Pathogenesis, Treatment and Prevention. Cape Town, South Africa 2009.

32. Fleiss $\mathrm{J}$ : Measuring nominal scale agreement among many raters Psychological Bulletin 1971, 76(5):378-382.

33. Lasko TA, Bhagwat JG, Zou KH, Ohno-Machado L: The use of receiver operating characteristic curves in biomedical informatics. Journal of Biomedical Informatics 2005, 38(5):404-415.

34. DeLong ER, DeLong DM, Clarke-Pearson DL: Comparing the Areas Under Two or More Correlated Receiver Operating Characteristics Curves: A Nonparametric Approach. Biometrics 1988, 44:837-845.

35. Tamura K, Nei M, Kumar S: Prospects for inferring very large phylogenies by using the neighbor-joining method. Proceedings of the National Academy of Sciences USA 2004, 101:11030-11035.

36. Edgar RC: MUSCLE: multiple sequence alignment with high accuracy and high throughput. Nucleic Acids Res 2004, 32(5):1792-1797.

37. Tamura K, Dudley J, Nei M, Kumar S: MEGA4: Molecular Evolutionary Genetics Analysis (MEGA) software version 4.0. Molecular Biology and Evolution 2007, 24:1596-1599.

38. R Development Core Team: R: A language and environment for statistical computing. R Foundation for Statistical Computing, Wien, Österreich. 2008 [http://www.R-project.org]. ISBN 3-900051-07-0

39. Harrigan PR: The influence of PCR amplification variation on the ability of population-based PCR to detect non-R5 HIV [abstract]. 8th European HIV Drug Resistance Workshop, Sorrento, Italy 2010.

40. German recommendations for determining HIV-1 coreceptor usage [http://www.viro.med.uni-erlangen.de/nrz/recommendation080324.pdf

41. Morand-Joubert L, Flandre P, Soulié C, Charpentier C, Desbois D, Ruffault A, Marcelin AG, Masquelier B, Calvez V, the ANRS AC11 Resistance Study Group: Evolution of coreceptor tropism and V3 loop resistance mutations in the blood cellular reservoir after introduction of maraviroc. XVIII HIV Drug Resistance Workshop, Fort Myers, Florida 2009.

42. Recordon-Pinson P, Descamps D, Soulie C, Lazrek M, Charpentier C, Montes B, Trabaud M-A, Cottalorda J, Amiel C, Morand-Joubert L, Tamalet 
C, Desbois D, Mace M, Ferre V, Vabret A, Ruffault A, Fleury H, Izopet J, BrunVezinet F, Marcelin AG, Reynes J, Flandre P, Calvez V, Masquelier B, ANRS AC11 Resistance Study Group: Genotypic prediction of HIV-1 tropism: correlation with the virological response to maraviroc and genotypic evolution on maraviroc therapy in the GenoTropism study. 12th European AIDS Conference (EACS), Cologne, Germany 2009.

43. Archer J, Braverman MS, Taillon BE, Desany B, James I, Harrigan PR, Lewis $M$, Robertson DL: Detection of low-frequency pretherapy chemokine (CXC motif) receptor 4 (CXCR4)-using HIV-1 with ultra-deep pyrosequencing. AIDS 2009, 23(10):1209-18.

44. Tsibris AM, Korber B, Arnaout R, Russ C, Lo CC, Leitner T, Gaschen B, Theiler J, Paredes R, Su Z, Hughes MD, Gulick RM, Greaves W, Coakley E, Flexner C, Nusbaum C, Kuritzkes DR: Quantitative deep sequencing reveals dynamic HIV-1 escape and large population shifts during CCR5 antagonist therapy in vivo. PLoS One 2009, 4(5):e5683.

doi: $10.1186 / 1742-4690-7-56$

Cite this article as: Prosperi et al., Comparative determination of HIV-1 COreceptor tropism by Enhanced Sensitivity Trofile, gp120 V3-loop RNA and DNA genotyping Retrovirology 2010, 7:56

Submit your next manuscript to BioMed Central and take full advantage of:

- Convenient online submission

- Thorough peer review

- No space constraints or color figure charges

- Immediate publication on acceptance

- Inclusion in PubMed, CAS, Scopus and Google Scholar

- Research which is freely available for redistribution

Submit your manuscript at www.biomedcentral.com/submit
C) Biomed Central 